\title{
Excision arthroplasty of the interphalangeal joint as an alternative to digit amputation in two dogs
}

Excision arthroplasty of the interphalangeal joint was used as an alternative to digit amputation for comminuted fractures of the phalangeal bones in two dogs. Both patients had return of pain-free use of the affected limb, even though both weight-bearing digits were involved in one case. This type of surgery might prove to be superior to the more invasive digit amputation, salvaging the digits and providing a better functional outcome.

H. De Rooster, M. Risselada AND H. vAN BREE*

Journal of Small Animal Practice (2007) 48, 169-173

DOI: $10.1111 /$ j.1748-5827.2006.00207.x

\section{INTRODUCTION}

Department of Medicine and Clinical Biology of Small Animals, and *Department of Medical Imaging and Orthopaedics, Faculty of Veterinary Medicine, University of Gent, Salisburylaan 133 9820 Merelbeke, Belgium
Phalangeal fractures are common in small animals (Anderson and others 1993, Probst and Millis 2003). In most cases, fractures of the distal extremities are caused by external trauma. Since there is little soft tissue cover, many fractures are open (Anderson and others 1993).

Management of phalangeal fractures is tailored to the individual patient. If minimal displacement is present, most fractures will heal with a minimum of intervention (Manley 1981, Anderson and others 1993). Surgical stabilisation might be warranted in high-performance animals when fragments are markedly displaced or when the fractures are intra-articular or open (Early and Dee 1980, Manley 1981, Dee and others 1990). However, adequate reconstruction of the fracture may not be possible because of the small size of the phalangeal bones or because of high comminution.

In selected cases, arthrodesis can be considered to restore the function of the digit by bony fusion of the joint surfaces (Moore and Withrow 1981). If repair methods fail, digit amputation offers an alternative in order to maintain functional and pain-free use of the limb, regardless of the intended use of the animal (Dee and others 1990, Anderson and others 1993, Piermattei and Flo 1997b).

This paper describes excision arthroplasty of the interphalangeal joint as an alternative to digital amputation in two dogs with multiple fractures of the phalan- ges. Excision arthroplasty of the metacarpophalangeal joint to treat osteoarthritis in three working dogs has been described previously in the German literature (Jauernig and others 1999). An excellent outcome was reported in two of the dogs, and a satisfying result was obtained in the remaining dog, which developed osteoarthritic changes in other metacarpophalangeal joints.

\section{CASE HISTORIES}

\section{Case 1}

A five-year-old, entire male Bernese Mountain dog was referred for evaluation of draining wounds at the level of the digits of the left hindleg. The dog had been involved in a dogfight a week before. The local practitioner attempted a primary closure on the day of the injury. At the time of referral, the dog had a body temperature of $38.5^{\circ} \mathrm{C}$ and an obvious enlargement of the left popliteal lymph node. On the left hind paw, necrotic and infected tissue was present on the dorsal aspect of the phalanges of the third and fourth digits, and the surrounding skin was inflamed. Bone fragments were visible within the wound. The nail of the second digit was split. There was a small skin defect and extensive inflammation.

Dorsoplantar and mediolateral radiographs were taken and revealed fractures of the first and second phalanges of the third and fourth digits (Fig 1). A double transverse fracture of the second phalanx, with several small fragments and severe medial displacement of the distal fragment, was seen in the third digit. A midtransverse fracture of the first phalanx was also observed in the same digit. In addition, these fragments were displaced dorsally. Soft tissue swelling was apparent as well.

In the area of the fourth digit, a transverse and slightly oblique fracture of the second phalanx with a fragment was observed, and there was moderate displacement of the distal fragment. A slightly 


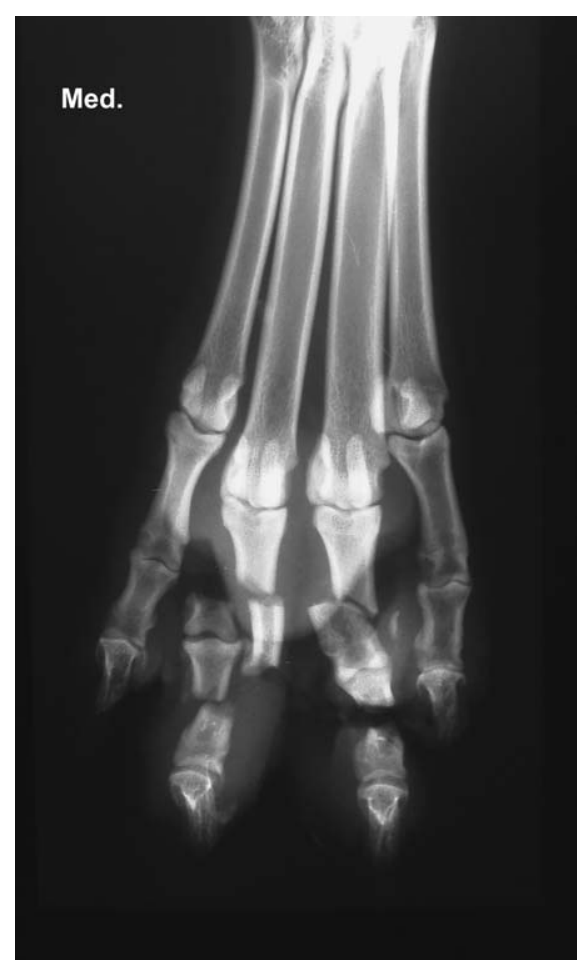

FIG 1. Preoperative radiographs revealed transverse fractures of both the first and second phalanx of the third and fourth digit (case 1, Bernese Mountain dog)

oblique fracture with distal displacement of the first phalanx was noticed as well. The dog was anaesthetised, analgesia was provided by $4 \mathrm{mg} / \mathrm{kg}$ intravenous carprofen (Rimadyl; Pfizer), and the wound was debrided. The distal third of the first phalanx of the third digit, attached to the proximal epiphysis of the second phalanx by the joint capsule and collateral ligaments, was visible in the wound. The fragments were completely detached from soft tissues and removed en bloc. The proximal fracture site was smoothed with a rongeur. At the level of the fourth digit, the diaphyseal fragment of the first phalanx was lying loose in the wound and taken out. The distal epiphysis of the first phalanx was also visible through the wound and excised after sharp dissection of the collateral ligaments and the joint capsule. The articular cartilage of the proximal end of the second phalanx was removed.

The more distal fracture was covered by healthy soft tissue, thus it was not visible through the wound and hence was left untouched. The surgical site was lavaged with sterile saline. Tissue was submitted for bacterial identification and antimicro- bial susceptibility. The wound was partially closed with non-absorbable suture material (polyamide, Ethilon; Ethicon) over a small Penrose drain.

After surgery, the foot was placed in a sterile padded bandage. Analgesia was continued by oral intake of $2 \mathrm{mg} / \mathrm{kg}$ carprofen (every 12 hours). A partially resistant Staphylococcus intermedius was isolated from the tissue specimen. Based on the antibacterial susceptibility pattern results received at day 3 , the $\mathrm{dog}$ was given $5 \mathrm{mg} / \mathrm{kg}$ enrofloxacin (Baytril; Bayer), orally (PO), every 24 hours, instead of $12.5 \mathrm{mg} / \mathrm{kg}$ amoxicillin and clavulanic acid (Synulox; Pfizer), PO, every 12 hours, for 10 days. The wound was flushed with sterile saline at least once a day and then wrapped in a sterile bandage. The drain was removed at day 6 , and the dog was discharged four days later.

By that time, the skin wound at the level of the fourth digit had almost healed completely; granulation tissue was forming at the medial aspect of the wound on the third digit. At a control visit two months later, the damaged nail of the second digit was pushing into the granulation tissue, causing an exudative inflammation of the nail bed. The owners reported that the dog had been running, playing, jumping and using stairs without any gait abnormality, but the persistent smell and discharge from the nail required further intervention.

Distal digit amputation was advised. However, due to a misunderstanding, the local practitioner amputated the second digit at the metatarsophalangeal level. On request, additional radiographs were taken after the surgical intervention and were sent to us (Fig 2). Minimal bony reactions were encountered at the resection sites. The dog was eventually euthanased by the local practitioner six months after the last surgery; he had been completely free of lameness, but he finally developed malignant histiocytosis. The owners declined a post-mortem examination.

\section{Case 2}

A five-month-old, entire male boxer was presented after being struck by a lawn mower. Physical examination of the dog revealed no abnormalities other than wounds on the right fore paw. The second digit had a skin laceration on the dorsal

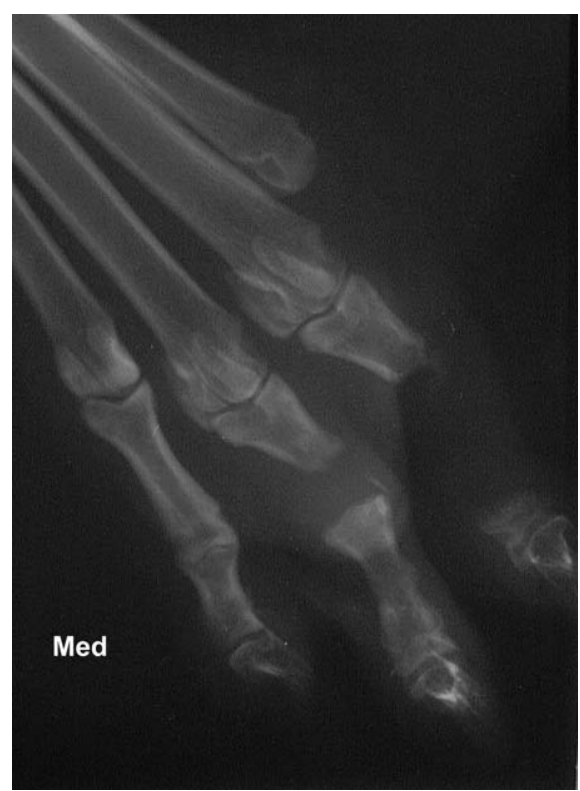

FIG 2. Postoperative radiographs (two-month follow-up) showed high digital amputation of the second digit and minimal bony reaction at the resection sites of the third and fourth digit (case 1, Bernese Mountain dog)

aspect of the first phalanx, and the underlying bone was visible through the wound. A comminuted open intra-articular fracture of the third digit was also present.

Preoperative dorsoplantar radiographs of the left metacarpal area confirmed fractures of the second and third digits (Fig 3). A transverse fracture with minimal displacement of the first phalanx of the second digit was observed. A comminuted fracture of the proximal part of the second phalanx of digit three - probably involving the interphalangeal joint space was also present, along with substantial soft tissue swelling. The dog was anaesthetised, $10 \mathrm{mg} / \mathrm{kg}$ intravenous amoxicillin (Clamoxyl; GlaxoSmithKline) and $2 \mathrm{mg} / \mathrm{kg}$ carprofen were administered, and the wounds were extensively flushed with sterile saline. The loose bone fragments at the level of the proximal interphalangeal joint of the third digit were removed, and the distal aspect of the first phalanx was removed with rongeurs. The minimally displaced transverse fracture of the second digit was left untouched. A vacuum drain was inserted. The soft tissue was apposed with fine resorbable suture material (poliglecaprone, Monocryl; Ethicon), and the skin was apposed using non-resorbable suture material (polyamide). 


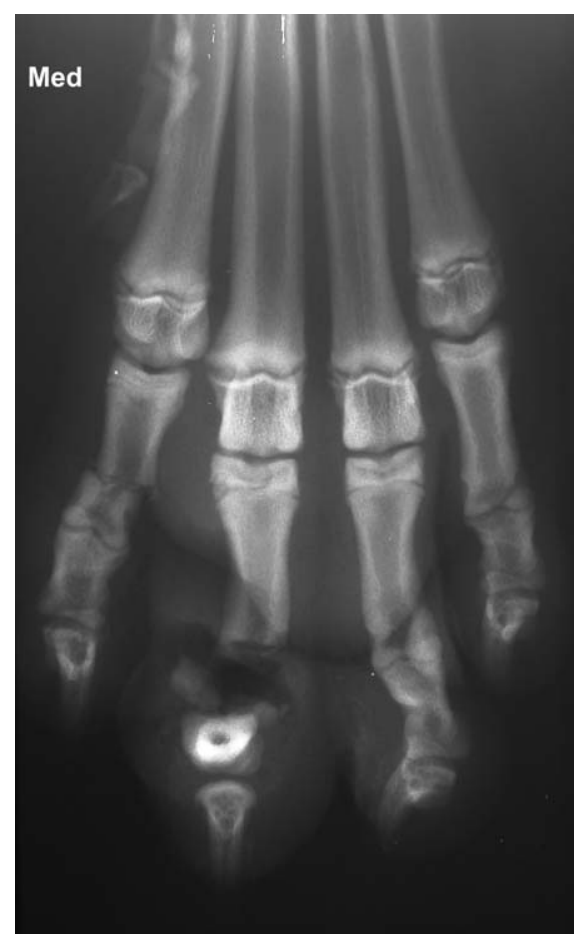

FIG 3. Preoperative radiographs confirmed a comminuted fracture of the proximal epiphysis of the second phalanx of the third digit and a transverse fracture of the first phalanx of the second digit (case 2 , boxer)

Postoperatively, a bandage was applied that was changed daily. The drain was removed three days postoperatively, and the dog was sent home on $2 \mathrm{mg} / \mathrm{kg}$ oral carprofen (every 12 hours) and $12.5 \mathrm{mg} / \mathrm{kg}$ amoxicillin and clavulanic acid (PO, every 12 hours) for seven days. The dog was fully weight-bearing after one week. Light bandages were applied to prevent selfmutilation. When the dog was re-examined three weeks postoperatively, the skin wounds had healed uneventfully. The dog showed no pain while walking or when the paw was manipulated.

During stance, however, the dog loaded the right metacarpal pad much more than the individual digital pads. At the owner's request, follow-up radiographs were taken (Fig 4). A non-bridging callus with new bone that was formed at the edges created by the excision arthroplasty was visible; no other abnormalities could be detected, and the fracture of the second digit had healed with minimal callus formation. No special treatment was instituted, and the abnormal loading subsided over the next month.

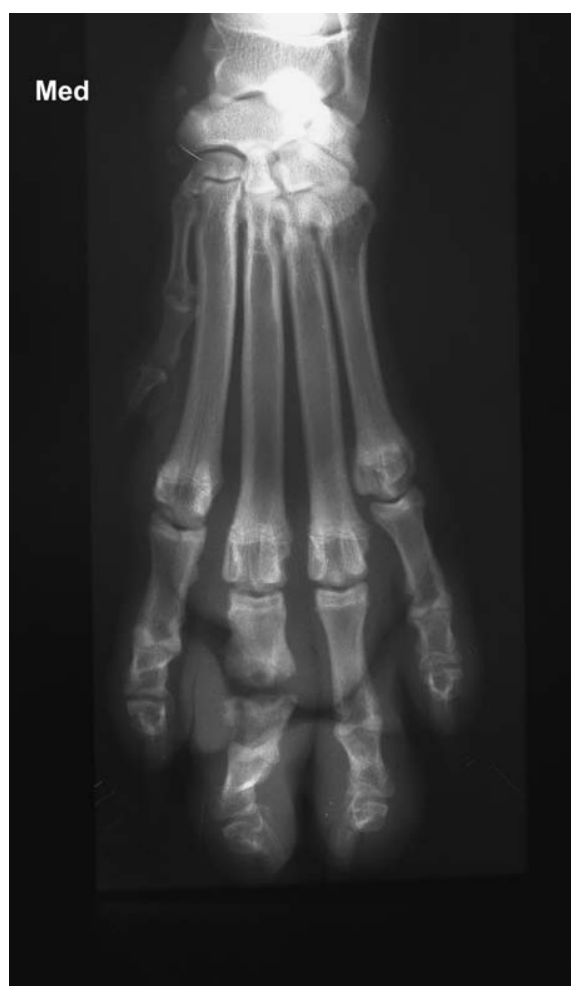

FIG 4. Postoperative radiographs (three-week follow-up) showed bony reaction at the resection site of the third digit and a healed fracture of the second digit (case 2 , boxer)

At 17 months after surgery, a physical examination of the dog was performed and follow-up radiographs were taken. The dog showed a perfectly normal gait at walk and trot. A small skin scar could be seen at the dorsal aspect of the second and third digits, but the angulation of all phalanges appeared normal at stance. Unexpectedly, the dorsoplantar and mediolateral radiographs showed a perfect ankylosis of the proximal interphalangeal joint of the third digit (Fig 5).

\section{DISCUSSION}

Different types of excisional arthroplasty in small animals have been described; the goal of this type of surgery is the creation of a functional pseudoarthrosis. By avoiding bone-to-bone contact, a pain-free repair is achieved. Excision arthroplasty is usually performed because of osteoarthritic joint changes; however, it is sometimes performed for intra-articular fractures as well.

Femoral head and neck excision is the best-known procedure in dogs, but the

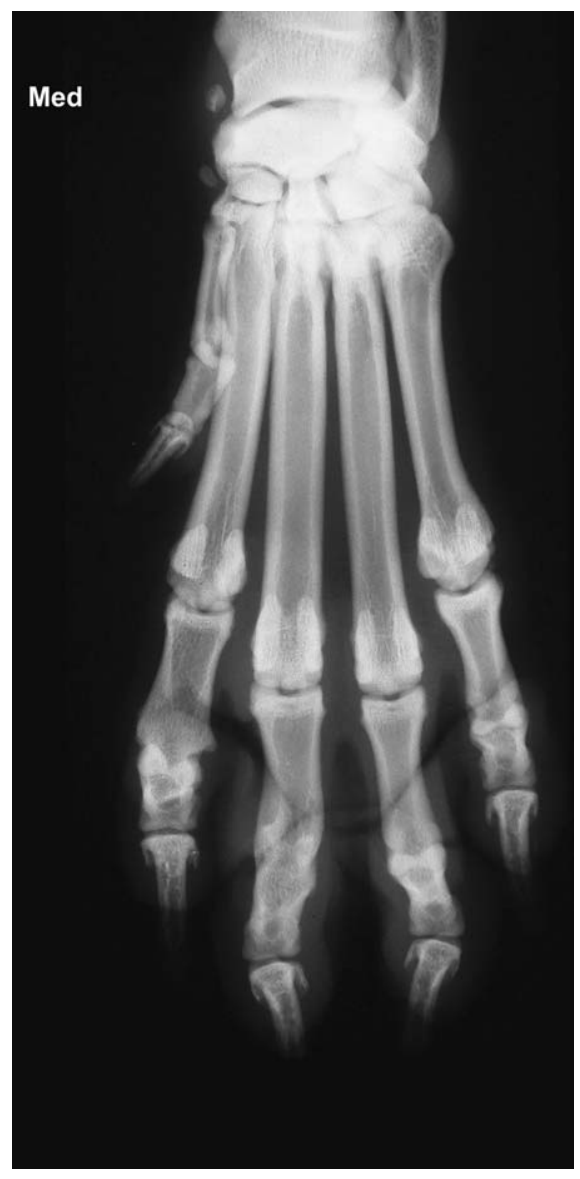

FIG 5. Postoperative radiographs (17-month follow-up) revealed an ankylosis of the proximal interphalangeal joint of the third digit (case 2, boxer)

shoulder and even the elbow are also amenable to excision arthroplasty (Berzon and others 1980, Breucker and Piermattei 1988, Franczuszki and Parkes 1988, Piermattei and Flo 1997a). One article has previously documented excision arthroplasty of the metacarpophalangeal joint as an alternative treatment to arthrodesis in three working dogs with chronic osteoarthritis (Jauernig and others 1999).

In human beings, excision arthroplasty of the first metatarsophalangeal joint of the big toe is a common operation used in low-demand patients to correct hallux rigidus, a stiffness of the main joint created by arthritis (Flamme and others 1998, Hamilton and Hubbard 2000, Thomas and others 2005). Proximal interphalangeal joint excisional arthroplasty is commonly performed to correct second hammertoe deformity, a dorsiflexion at the metatarsophalangeal joint combined 
with a plantarflexion at the proximal interphalangeal joint of the second toe (O'Kane and Kilmartin 2005).

The two cases of excisional arthroplasty of the interphalangeal joint in the current report had an excellent long-term outcome. Case 2 temporarily showed an unusual placement of the foot. It is not likely that this was related to flexor or extensor tendon pathology since the whole foot (and not just the operated digit) was involved. One might assume that the abnormal foot placement was due to digital discomfort, although the dog did not show any pain on passive manipulation. Although floating toe is reported postoperatively in human beings, the fact that the pulp of the toe does not contact the ground is almost certainly a consequence of existing damage or instability of the metatarsophalangeal joint rather than a true complication of excisional arthroplasty of the interphalangeal joint. Hyperextension of the operated toe occurred in only 1 per cent of the human cases (O'Kane and Kilmartin 2005). Other than that, no serious complications are encountered after excisional arthroplasty of the proximal interphalangeal joint in human beings and an excellent long-term outcome is achieved (O'Kane and Kilmartin 2005).

In the two cases of excisional arthroplasty reported here, no attempts were made to interpose soft tissues between the cut surfaces. Although some reports indicate that better results are obtained by interposing soft tissue between the bone surfaces, there is no proof of any benefit associated with placement of soft tissue between the femur and the acetabulum after femoral head and neck excision (Mann and others 1987, Harasen 2004). In fact, interpositional grafts may even create additional morbidity and complications (Lewis and others 1988). Retrospectively, it can be questioned whether interposition of soft tissues would have prevented the bone union in case 2 . This dog was still immature at the time of surgery, so its young age might explain the formation of a bridging callus instead of a pseudoarthrosis. To our knowledge, bone union is not a reported complication after excisional arthroplasty of the phalangeal joints in human beings, but, only older patients undergo this type of surgery because hallux rigidus and second hammertoe deformities are both conditions encountered at advanced age. In the veterinary literature, the formation of a synostosis between the first and second phalanx has been reported as a complication in an old dog undergoing transarticular external skeletal fixator fixation for the treatment of proximal interphalangeal instability (Guilliard 2003).

Digit amputation is frequently performed in small animals. It is unquestionably necessary when malignant disease is suspected (Bateman 1960). In severely comminuted fractures of the phalanges, or in cases of severe ligamentous injuries, digit amputation is a good option, even in performing animals (Anderson and others 1993). However, amputation might be over-used for conditions that are in some way reparable (Bateman 1960, Sweeney 1998). The more distal the amputation, the better the prognosis. Amputation of the second or fifth digit at any level has a good result in most dogs, but the prognosis is poorer in the weight-bearing digits (Piermattei and Flo 1997b). At the moment of forward thrust, the dog's entire bodyweight is on the two middle digits of one foot (Bateman 1960). Therefore, if the main weight-bearing digits are affected (as was the case in both dogs described here), alternative measures should certainly be considered. Some surgeons have modified the standard digit amputation technique by removing the bones while saving the pad (Early and Dee 1980, Dee and others 1990, Basher 1994). The advantages of excision arthroplasty over amputation are the maintenance of some functional use of the affected digits and an undisturbed cosmetic appearance.

In the treatment of fractures, it is generally accepted that bone fragments with soft tissue attachments can be preserved. Bone chips from open fractures, however, should be removed as they may prevent complete granulation (Pead and LangleyHobbs 1999). During the debridement of the wounds, only fragments of bone that were within the primary defect were removed. The minimally displaced phalangeal fractures that were covered by healthy soft tissue healed in both dogs, with minimal callus formation, although no specific attention was given to external coaptation.
Arthrodesis might be a viable alternative, but it requires internal fixation devices and cancellous bone grafting. Attempts to arthrodese the proximal interphalangeal joint have been reported to be successful in the front limbs, but not in the hindlimbs, of high-performance dogs (Dee and others 1990). Arthrodesis of the metatarsophalangeal joints of two load-bearing digits has been well tolerated in a large dog (Van Ee and Blass 1989).

\section{Conclusions}

Excisional arthroplasty could present as a reasonable alternative for many patients who are candidates for phalangeal amputation. The procedure might be suitable for any condition in which the integrity of the interphalangeal joints has been compromised and primary repair is not feasible. However, our sample of two dogs is far too small to detail all the indications and complications of excision arthroplasty of the interphalangeal joints in dogs. Excision arthroplasty of the metacarpophalangeal/metatarsophalangeal joints and of the interphalangeal joints should be further examined as a treatment option for open fractures invading the joint, salvage after failed intra-articular fixation, cases where infection is present and cases of severe osteoarthritis. Long-term radiographs should be taken to document whether a pseudoarthrosis or an osseous fusion will be the final outcome.

\section{References}

Anderson, M. A., Payne, J. T. \& Constantinescu, G. M. (1993) Managing fractures and related injuries of the distal extremities in dogs and cats. Veterinary Medicine 88, 957-968

Basher, A. (1994) Foot injuries in dogs and cats. Compendium on Continuing Education for the Practicing Veterinarian 16, 1159-1176

Bateman, J. K. (1960) The racing greyhound. Veterinary Record 72, 893-897

Berzon, J. L., Howard, P. E., Covell, S. J., Trotter, E. J. \& DuelAND, R. T. (1980) A retrospective study of the efficacy of femoral head and neck excisions in 94 dogs and cats. Veterinary Surgery $\mathbf{9}, 88-92$

Breucker, K. A. \& Piermattel, D. L. (1988) Excision arthroplasty of the canine scapulohumeral joint: report of three cases. Veterinary Comparative Orthopedics and Traumatology 3, 134-140

Dee, J. F., Dee, L. G. \& Eaton-Wells, R. D. (1990) Injuries of high performance dogs. In: Canine Orthopedics. 2nd edn. Ed W. G. Whittick. Lea \& Febiger, Philadelphia, PA, USA. pp 519-570

EARLY, T. D. \& DEE, J. F. (1980) Trauma to the carpus, tarsus, and phalanges of dogs and cats. Veterinary Clinics of North America: Small Animal Practice 10, 717-747

Flamme, C. H., Wulker, N., Kuckerts, K., Gosse, F. \& WIRTH, C. J. (1998) Follow-up results 17 years after resection arthroplasty of the great toe. 
Archives of Orthopaedic and Trauma Surgery 117, 457-460

FranczuszkI, D. \& Parkes, L. J. (1988) Glenoid excision as a treatment in chronic shoulder disabilities: surgical technique and clinical results. Journal of the American Animal Hospital Association 24, 637-643

GuILLIARD, M. J. (2003) Proximal interphalangeal joint instability in the dog. Journal of Small Animal Practice 44, 399-403

Hamilton, W. G. \& Hubbard, C. E. (2000) Hallux rigidus. Excisional arthroplasty. Foot Ankle Clinics 5, 663-671

Harasen, G. (2004) The femoral head and neck ostec tomy. Canadian Veterinary Journal 45, 163-164 Jauernig, S., Spreng, D. \& Schawalder, P. (1999) Die Exzisionsarthroplastik als Therapie der rezidivierenden Osteoarthritis der Zehengelenke des Hundes. Schweizer Archiv für Tierheilkunde 141 461-468

Lewis, D. D., Bellah, J. R., McGavin, M. D. \& Gaskin, J. M. (1988) Postoperative examination of the biceps femoris muscle sling used in excision of the femoral head and neck in dogs. Veterinary Surgery 17, 269-277
Manley, P. A. (1981) Distal extremity fractures in small animals. Journal of Veterinary Orthopedics 2, 38-48

Mann, F. A., Tangner, C. H., Wagner-Mann, C., Read, W. K., Hulse, D. A., Puglisi, T. A. \& Hobson, H. P. (1987) A comparison of standard femoral head and neck excision and femoral head and neck excision using a biceps femoris muscle flap in the dog. Veterinary Surgery 16, 223-230

Moore, R. W. \& Withrow, S. J. (1981) Arthrodesis. Compendium on Continuing Education for the Practicing Veterinarian 3, 319-330

O'KANE, C. \& KILMARTIN, T. (2005) Review of proximal interphalangeal joint excisional arthroplasty for the correction of second hammer toe deformity in 100 cases. Foot \& Ankle International 26, 320-325

PeAd, M. \& Langley-HobBs, S. (1999) Acute management of orthopaedic and external soft tissue injuries. In: BSAVA Manual of Canine and Feline Emergency and Critical Care. Eds L. King and R. Hammond. British Small Animal Veterinary Association, Gloucester, UK. pp 191-199

Piermattel, D. L. \& Flo, G. L. (1997a) The elbow joint. In: Brinker, Piermattei, and Flo's Handbook of
Small Animal Orthopedics and Fracture Repair. 3rd edn. Eds D. L. Piermattei and G. L. Flo. W. B. Saunders, Philadelphia, PA, USA. pp 288 320

Piermattei, D. L. \& Flo, G. L. (1997b) Fractures and other orthopedic conditions of the carpus, metacarpus, and phalanges. In: Brinker, Piermattei, and Flo's Handbook of Small Animal Orthopedics and Fracture Repair. Brd edn. Eds D. L. Piermatte and G. L. Flo. W. B. Saunders, Philadelphia, PA, USA. pp 344-389

Probst, C. W. \& Miluss, D. L. (2003) Carpus and digits. In: Textbook of Small Animal Surgery. 3rd edn. Ed D. Slatter. W. B. Saunders, Philadelphia, PA, USA. pp 1974-1988

SweEneY, P. A. (1998) Digit amputation in dogs. Veterinary Record 143, 540

Thomas, S., Kinninmonth, A. W. G. \& Senthil Kumar, C. (2005) Long-term results of the modified Hoffman procedure in the rheumatoid forefoot. Journal of Bone and Joint Surgery (Am) 87-A, 748-752

VAN EE, R. T. \& Blass, C. E. (1989) Arthrodesis of metatarsophalangeal joints in a dog. Journal of the American Veterinary Medicine Association 194, $82-84$ 